\title{
Inhibition of farnesyl pyrophosphate synthase attenuates angiotensin II-induced fibrotic responses in vascular smooth muscle cells
}

\author{
CHANG-QING DU, XIAO-WEI LIU, GUANG-ZHONG ZENG, HONG-FENG JIN and LI-JIANG TANG \\ Department of Cardiology, Zhejiang Hospital, Hangzhou, Zhejiang 310013, P.R. China
}

Received January 28, 2015; Accepted March 26, 2015

DOI: $10.3892 /$ ijmm.2015.2166

\begin{abstract}
Through the regulation of the RhoA/Rho kinase (ROCK) pathway, angiotensin II (Ang II)-induced fibrotic responses contribute to vascular remodeling. Farnesyl pyrophosphate synthase (FPPS) plays an important role in cardiovascular remodeling through the modulation of the above-mentioned pathway. However, the role of FPPS in Ang II-induced fibrotic responses and the related molecular mechanisms have not yet been elucidated. In the present study, vascular smooth muscle cells (VSMCs) from Sprague-Dawley (SD) rats were stimulated with Ang II. Cell proliferation was measusred usin the cell counting kit-8 (CCK-8). The levels of connective tissue growth factor (CTGF), FPPS, and those of phosphorylated and total extracellular signal-regulated kinase (ERK)1/2, p38 and c-Jun N-terminal kinase (JNK) were determined by western blot analysis. RhoA activity was determined using a pull-down assay. The results revealed that stimulation with Ang II enhanced cell proliferation, and increased the protein expression levels of FPPS and CTGF in the VSMCs. The inhibition of FPPS with ibandronate sodium attenuated the Ang II-induced increase in cell proliferation, CTGF expresison and RhoA activity; these effects were partially reversed by treatment with geranylgera-
\end{abstract}

Correspondence to: Dr Chang-Qing Du, Department of Cardiology, Zhejiang Hospital, 12 Lingying Road, Hangzhou, Zhejiang 310013, P.R. China

E-mail: ddcq82@hotmail.com

Abbreviations: VSMCs, vascular smooth muscle cells; SHR, spontaneously hypertensive rats; Ang II, angiotensin II; FPP, farnesyl pyrophosphate; GGPP, geranylgeranyl pyrophosphate; GGOH, geranylgeraniol; FOH, farnesol; Iban, ibandronate sodium; CTGF, connective tissue growth factor; FPPS, farnesyl pyrophosphate synthase; ECM, extracellular matrix; MAPK, mitogen-activated protein kinase; DMEM, Dulbecco's modified Eagle's medium; FBS, fetal bovine serum; SDS-PAGE, sodium dodecyl sulphate-polyacrylamide gel electrophoresis; ERK, extracellular signal-regulated kinase; JNK, c-Jun N-terminal kinase; GAPDH, glyceraldehyde-3phosphate dehydrogenase

Key words: farnesyl pyrophosphate synthase, angiotensin II, vascular smooth muscle cells, connective tissue growth factor, proliferation, mitogen-activated protein kinase niol and were mimicked by GGTI-286. Furthermore, both SB203580 (a specific inhibitor of p38) and SP600125 (JNK1, JNK2 and JNK3 inhibitor) diminished the Ang II-induced production of CTGF; however, the inhibition of FPPS reduced the Ang II-induced activation of p38 mitogen-activated protein kinase (MAPK) and JNK. In conclusion, our data indicate that FPPS may play an important role in Ang II-induced fibrotic responses in VSMCs, and the underlying mechanisms at least partly involve the modulation of RhoA activity, and the p38 and JNK pathways.

\section{Introduction}

Vascular remodeling, characterized by endothelial dysfunction, vascular smooth muscle cell (VSMC) proliferation and by the excessive accumulation of extracellular matrix (ECM), plays an important role in the development of various cardiovascular diseases, including hypertension, atherosclerosis and restenosis following balloon injury (1,2). Angiotensin II (Ang II), the main effector peptide of the renin-angiotensin system, plays a critical role in the development of vascular remodeling. Ang II-induced VSMC proliferation increaseS the production of profibrotic factors, such as connective tissue growth factor (CTGF) (3). CTGF has been demonstrated as a strong profibrogenic factor in vascular remodeling through the regulation of VSMC proliferation and migration (4). In addition, CTGF has been shown to be overexpressed in human atherosclerotic lesions (5) and in the aorta of of Ang II-infused rats and cultured VSMCs (6).

The molecular mechanisms involved in Ang II-induced vascular remodeling, include the activation of several intracellular signaling systems, such as mitogen-activated protein kinases (MAPKs), redox processes and RhoA/Rho kinase (ROCK) (7). Accumulating evidence has demonstrated that Rho/ROCK are critically involved in Ang II-induced vascular remodeling $(8,9)$. Thus, the activation of Rho/ROCK represents one of the key signal transduction pathways that mediate the pathophysiological functions of Ang II. Howerver, the molecular mechanisms through which Ang II activates the Rho/ ROCK pathway have not yet been fully elucidated.

Farnesyl pyrophosphate synthase (FPPS) plays an important role in the mevalonate pathway. It catalyzes isopentenyl pyrophosphate and dimethylallyl pyrophosphate to form geranylgeranyl pyrophosphate (GGPP) and farnesyl pyrophosphate (FPP), and regulates the small GTPase-binding proteins, 
including Ras and Rho which have been implicated in the pathogenesis of various cardiovascular diseases (10). Recent studies have indicated that FPPS participates in the pathogenesis of cardiovascular remodeling through the modulation of the RhoA/ROCK and MAPK pathways (11-15). In a recent previous study of ours, we demonstrated that the inhibition of FPPS prevented norepinephrine (NE)-induced fibrotic responses in VSMCs obtained from spontaneously hypertensive rats (SHRs) and that the underlying mechanisms involved the Ras kinase and p38 pathways (16). However, the role of FPPS in Ang II-induced fibrotic responses and the related molecular mechanisms have not yet been elucicated. Thus, the aim of the present study was to investigate the molecular mechanisms involved in the Ang II-induced production of CTGF in the VSMCs associated with vascular remodeling. We wished to examine the hypothesis that FPPS may be a potent regulator of the Ang II-RhoA/ Rho-kinase (ROCK) pathway and CTGF functions.

\section{Materials and methods}

Materials. Ang II, ibandronte sodium (Iban), FTI-276 (a selective inhibitor of farnesyltransferase), GGTI-286 [a selective inhibitor of geranylgeranyl transferase-I (GGTase I)], farnesol $(\mathrm{FOH})$, geranylgeraniol $(\mathrm{GGOH})$, p38 MAPK inhibitor (SB203580), extracellular signal-regulated kinase (ERK)1/2 inhibitor (PD98059) and c-Jun N-terminal kinase (JNK) inhibitor (SP600125) were purchased from Sigma (St. Louis, MO, USA). Dulbecco's modified Eagle's medium (DMEM), penicillin-streptomycin and fetal bovine serum (FBS) and all other cell culture reagents were purchased from Gibco (Life Technologies, Carlsbad, CA, USA). Unless otherwise stated, the remaining agents used for cell treatment were prepared in sterile saline and diluted to a working concentration in DMEM. Rabbit anti-CTGF polyclonal antibody (ab6992) and rabbit anti-FPPS polyclonal antibody (ab153805) were obtained from Abcam (Cambridge, UK). MAPK antibodies, including phosphorylated ERK1/2 (p-ERK1/2; 4370), ERK1/2 (4695), phosphorylated p38 (p-p38; 4511) and p38 (8690) were obtained from Cell Signaling Technology (Danvers, MA, USA). Phosphorylated JNK1/2/3 (p-JNK1/2/3; 3893-1) and JNK1 (3496-1) antibodies were obtained from Epitomics (Burlingame, CA, USA). The RhoA activation assay kit was purchased from Cytoskeleton, Inc. (Denver, CO, USA). Enhanced chemiluminescence (ECL) reagent was from Amersham International (Bucks, UK).

Specific pathogen-free male Sprague-Dawley (SD) rats, 4-6 weeks of age, were obtained from the Experimental Animal Center, Zhejiang Academy of Sciences (Hangzhou, China). All procedures were performed in accordance with the revised 1996 National Institutes of Health Guidelines for the Care and Use of Laboratory Animals (NIH Publication no. 85-23) and the Animal Care Committee of Zhejiang Hospital, Zhejiang, China.

VSMC culture and treatment. Primary cultures of VSMCs were isolated from the thoracic aortas of SD rats as previously described using the collagenase method $(6,16)$. The cells were incubated in a humidified incubator at $37^{\circ} \mathrm{C}$ and $5 \% \mathrm{CO}_{2}$. Cells between passages 3 and 5 were used in all the experiments. Cell quiescence was established by first transferring the cells to 9 -well culture plates at $80 \%$ confluence followed by maintenance in quiescence medium consisting of DMEM with $0.1 \% \mathrm{FBS}$ for $48 \mathrm{~h}$. The quiescent cells were treated in the presence or absence of Ang II at $0.1 \mu \mathrm{M}$ for $24 \mathrm{~h}$ as previously described (6,7). In some experiments, the cells were preincubated for $2 \mathrm{~h}$ with Iban $10 \mu \mathrm{M}$, Iban plus GGOH $(30 \mu \mathrm{M})$, Iban plus FOH $(30 \mu \mathrm{M})$, GGTI-286 (a selective inhibitor of GGTase I, $10 \mu \mathrm{M}$ ) or FTI-276 (a selective inhibitor of farnesyltransferase, $10 \mu \mathrm{M})$. Furthermore, in some other experiments, in order to inhibit the MAPK pathway, PD98059 (an ERK1/2 inhibitor, $50 \mu \mathrm{M}$ ), SB203580 (a p38 MAPK inhibitor, $10 \mu \mathrm{M}$ ) and SP600125 (a JNK1/2/3 inhibitor, $10 \mu \mathrm{M}$ ) were added to the cells $2 \mathrm{~h}$ prior to stimulation with Ang II. All the agents mentioned above were maintained in quiescence medium.

Cell proliferation assay. VSMC proliferation was measured using a by cell counting kit (CCK-8) (Dojindo Laboratories, Tokyo, Japan) as previously described (16) and according to the manufacturer's instructions.

The VSMCs were transferred to a 96-well plate at a density of $1 \times 10^{4}$ cells/well. After $24 \mathrm{~h}$ of treatment, $10 \mu \mathrm{l} \mathrm{CCK-8} \mathrm{solu-}$ tion were added to each well followed by incubation at $37^{\circ} \mathrm{C}$ for an additional $2 \mathrm{~h}$. The absorbance of each well at a wavelength of $450 \mathrm{~nm}$ was then measured using the Bio-Rad 680 microplate reader (Bio-Rad, Hercules, CA, USA). Cell proliferation was expressed as the optical density.

Western blot analysis. Cellular protein was isolated by homogenization with cell lysis buffer. The protein concentration was then determined using the BCA method. Equal amounts of protein were denatured and subjected to $10 \%$ sodium dodecyl sulphate-polyacrylamide gel electrophoresis (SDS-PAGE). The membranes were incubated with primary antibodies (against CTGF, FPPS, and phosphorylated and total ERK1/2, p38 and JNK) overnight at $4^{\circ} \mathrm{C}$. Following incubation with secondary antibodies (HRP-labeled goat anti-rabbit: Cat. no. GAR007; MultiSciences Biotech Co., Ltd, Hangzhou, China) for $1 \mathrm{~h}$ at room temperature and washing with TBST, the proteins were detected using an ECL Plus system (Amersham Bioscience, Piscataway, NJ, USA). To ensure equal protein loading, glyceraldehyde-3-phosphate dehydrogenase (GAPDH) was used as an internal control. The results of CTGF production were obtained from densitometric analysis and expressed as the ratio CTGF/GAPDH as n-fold over the control.

RhoA activation assay. RhoA activity was determined using a pull-down assay according to the manufacturer's instructions. In brief, a BCA protein assay was performed to equalize the total protein concentration. RBD-bound Rho from the cell lysates was 'pulled down' using agarose-conjugated Rhotekin-RBD and detected by western blot analysis using specific anti-RhoA antibody [Cat. no. ARH03, contained in the Rho Activation assay kit (BK036)]. A total of $20 \mu \mathrm{g}$ of total cell lysate per sample was used to detect the total amount of RhoA expression. In addition, GAPDH protein was used as an endogenous control.

Statistical analysis. The results are presented as the means \pm SEM and expressed as the n-fold increase over the controls. One-way analysis of variance (ANOVA) followed by the Bonferroni post hoc test were used to determine significant 

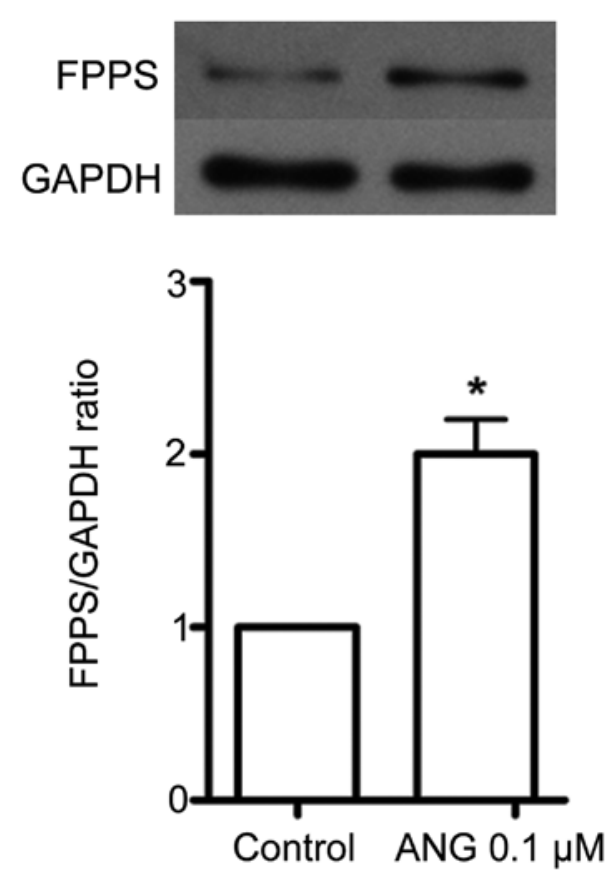

Figure 1. Protein expression of farnesyl pyrophosphate synthase (FPPS) in vascular smooth muscle cells (VSMCs) from SD rats before and after stimulation with angiotensin II (Ang II) . Quiescent VSMCs were incubated with Ang II $0.1 \mu \mathrm{M}$ for $24 \mathrm{~h}$. The protein levels of FPPS were determined by western blot analysis. The ratio of FPPS protein is set to ' 1 ' for the control group. Data are expressed as the means \pm SEM $(n=5)$. ${ }^{*} \mathrm{P}<0.05$ vs. the control group.

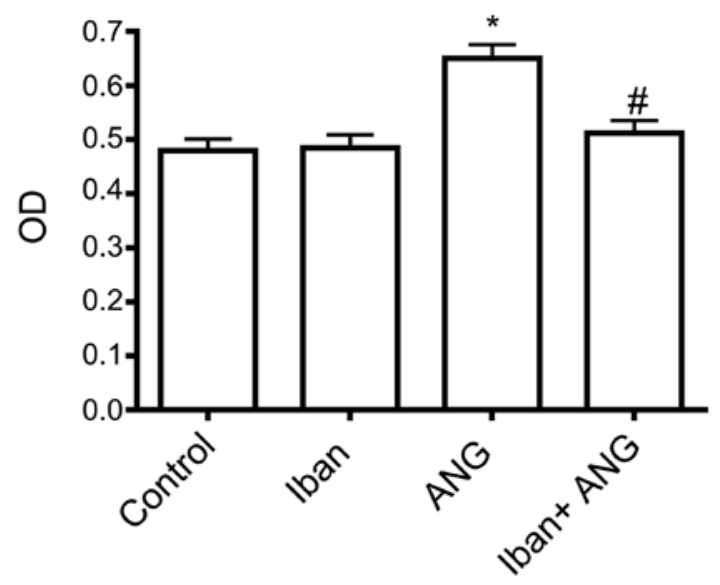

Figure 2. Ibandronate decreases vascular smooth muscle cell (VSMC) proliferation. Cell proliferation is expressed as the optical density which was determined using the cell counting kit-8 (CCK-8). Data are expressed as the means \pm SEM $(n=5)$. ${ }^{*} \mathrm{P}<0.05$ vs. the control group; ${ }^{"} \mathrm{P}<0.05$ vs. treatment with angiotensin II (Ang II),

differences among multiple groups. The results were considered statistically significant at a value of $\mathrm{P}<0.05$.

\section{Results}

Effects of Ang II on FPPS expression in cultured VSMCs. In the growth-arrested VSMCs, incubation with Ang II $(0.1 \mu \mathrm{M})$ for $24 \mathrm{~h}$ increased the protein expression of FPPS (P<0.05; Fig. 1).

Inhibition of FPPS decreases the fibrotic responses elicited by Ang II in cultured VSMCs. To investigate the potential

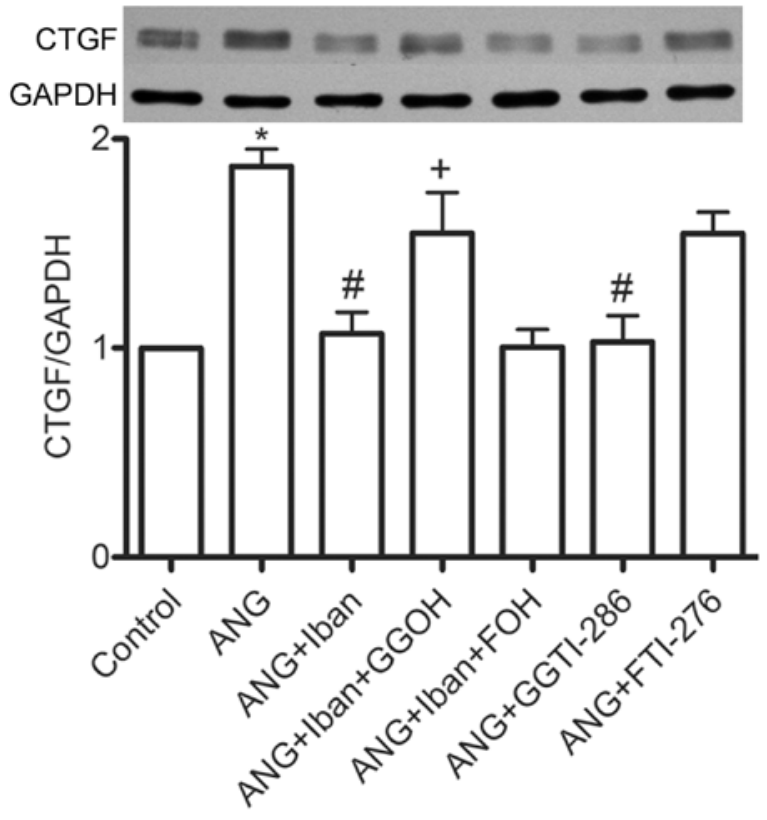

Figure 3. Ibandronate sodium (Iban) decreases connective tissue growth factor (CTGF) protein expression. The ant-fibrotic effects of Iban may be mediated through the inhibition of geranylgeranylated proteins. Quiescent vascular smooth muscle cells (VSMCs) were pre-incubated with Iban $(10 \mu \mathrm{M})$, GGTI286 (a selective inhibitor of geranylgeranyl transferase-I; $10 \mu \mathrm{M}$ ) or FTI-276 (a selective inhibitor of farnesyltransferase; $10 \mu \mathrm{M}$ ) for $2 \mathrm{~h}$ and then incubated with or without angiotensin II (Ang II) $0.1 \mu \mathrm{M}$ for $24 \mathrm{~h}$. Some cells were also incubated with geranylgeraniol $(\mathrm{GGOH} ; 30 \mu \mathrm{M})$ or farnesol $(\mathrm{FOH} ; 30 \mu \mathrm{M}) 2 \mathrm{~h}$ prior to the addition of the Iban. The results of the analysis of total connective tissue growth factor (CTGF) production were obtained frombydensitometric analysis and expressed as the ratio CTGF/glyceraldehyde-3-phosphate dehydrogenase (GAPDH) as n-fold over the control. Top panel shows a representative western blot and bottom panel shows the data of total CTGF production, presented as the means \pm SEM of 3 independent experiments. The ratio of CTGF is set to ' 1 ' for the control group. " $\mathrm{P}<0.05$ vs. control; ${ }^{\text {"}} \mathrm{P}<0.05$ vs. treatment with Ang II; ${ }^{+} \mathrm{P}<0.05$ vs. treatment with Ang II + Iban.

role of FPPS in the fibrotic responses elicited by Ang II, Iban, an FPPS inhibitor, was tested using the Ang II-stimulated VSMCs. Pre-incubation with $10 \mu \mathrm{M}$ Iban for $2 \mathrm{~h}$ reduced the Ang II-induced increase in cell proliferation and CTGF protein expression (Figs. 2 and 3). The addition of GGOH, but not that of $\mathrm{FOH}$, partly reversed the inhibitory effects of Iban on the expression of CTGF (Fig. 3). In addition, pre-treatment with GGTI-286, but not with FTI-276, substantially reduced the Ang II-induced incraase in the expression of CTGF (Fig. 3). These results suggest that Rho signaling is involved in this process.

Inhibition of FPPS modulates the Ang II-induced RhoA activation in cultured VSMCs. Stimulation with $0.1 \mu \mathrm{M}$ Ang II for $15 \mathrm{~min}$ increased the GTP-bound RhoA levels, as shown by pull-down assays (Fig. 4), which is in accordance with the findings of a previous study (7). We then wished to determine whether Iban inhibits the activation of RhoA induced by Ang II. Pre-treatment with $10 \mu \mathrm{M}$ Iban for $24 \mathrm{~h}$ diminished the Ang II-induced activation of RhoA, as shown by pull-down assays (Fig. 4). These data demonstrate that Iban inhibits RhoA activation induced by Ang II.

Inhibition of FPPS modulates the Ang II-induced MAPK activation in cultured VSMCs. Treatment with Ang II $0.1 \mu \mathrm{M}$ for 

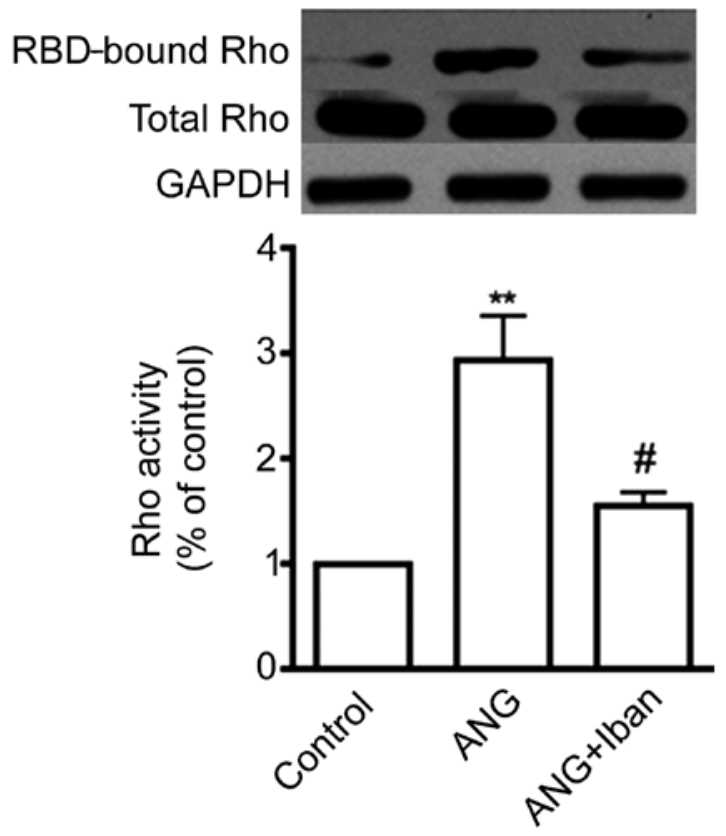

Figure 4. Ibandronate sodium (Iban) diminishes RhoA activation in angiotensin II (Ang II)-stimulated vascular smooth muscle cells (VSMCs). Cells were pre-treated for $24 \mathrm{~h}$ with Iban $10 \mu \mathrm{M}$ and then stimulated with Ang II $0.1 \mu \mathrm{M}$ for $30 \mathrm{~min}$. RhoA activity was determined by pull-down assays. Top panel shows a representative western blot and bottom panel shows data, presented as the means \pm SEM of 3 independent experiments. As a loading control, total RhoA and glyceraldehyde-3-phosphate dehydrogenase (GAPDH) expression levels were evaluated in the total cell lysates. The ratio of active RhoA is set to ' 1 ' for the control group. ${ }^{\text {** }} \mathrm{P}<0.01$ vs. control; " $\mathrm{P}<0.05$ vs. treatment with Ang II.

15 min triggered the phosphorylation of p38, JNK and ERK1/2 in the VSMCs, which is in accordance with the findings of a previous study (7). To investigate whether the activation of all 3 MAPKs is involved in the molecular mechanisms underlying the ability of Iban to suppress the Ang II-induced increase in the expression of CTGF, the VSMCs were treated with specific inhibitors of MAPKs (SB203580 for p38, PD98059 for ERK1/2 and SP600125 for JNK) pior to stimulation with Ang II. Both the p38 inhibitor and JNK inhibitor diminished the Ang II-induced production of CTGF (Fig. 5). Furthermore, pre-incubation with Iban inhibited the Ang II-induced activation of p38 and JNK, but not that of ERK1/2 (Fig. 6), suggesting that the activation of p38 and JNK is involved in the downregulation of CTGF by Iban. These data indicate that the inhibition of FPPS modulates the Ang II-induced production of CTGF through the regulation of the p38 MAPK and JNK pathways in cultured VSMCs.

\section{Discussion}

In the present study, we demonstrated that FPPS expression is induced by Ang II in cultured VSMCs from SD rats. Iban, an FPPS inhibitor, inhibited the Ang II-induced cell proliferation and the production of CTGF in the cultured VSMCs. The underlying mechanisms involved the inhibition of RhoA signaling and the modulation of the p38 MAPK and JNK pathways.

Through the inhibition of osteoclastic activity and bone resorption, nitrogen-containing bisphosphonates (N-BPs) including alendronate, Iban and zoledronate are commonly used for the treatment of bone-related diseases in clinical
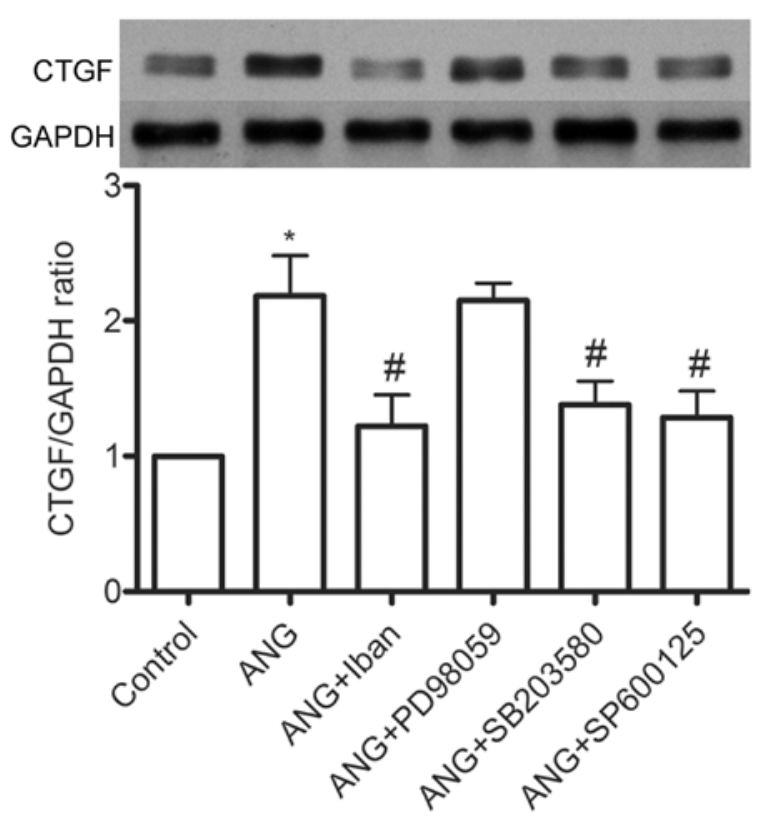

Figure 5. Inhibition of p38 mitogen-activated protein kinase (MAPK) and c-Jun N-terminal kinase (JNK) diminishes the angiotensin II (Ang II)-induced production of connective tissue growth factor (CTGF) in vascular smooth muscle cells (VSMCs). VSMCs were incubated for $2 \mathrm{~h}$ with $10 \mu \mathrm{M}$ Iban [farnesyl pyrophosphate synthase (FPPS) inhibitor], $10 \mu \mathrm{M}$ SB203580 (p38 inhibitor), $50 \mu \mathrm{M}$ PD98059 [extracellular signal-regulated kinase (ERK)-1/2 inhibitor] or $10 \mu \mathrm{M} \mathrm{SP600125} \mathrm{(JNK} \mathrm{inhibitor)} \mathrm{prior} \mathrm{to} \mathrm{stimulation} \mathrm{with} 0.1 \mu \mathrm{M}$ Ang II for $24 \mathrm{~h}$. Top panel shows a representative western blot and bottom panel shows data, presented as the means \pm SEM of 3 independent experiments. The ratio of CTGF is set to ' 1 ' for control group. ${ }^{*} \mathrm{P}<0.05$ vs. control; ${ }^{\#} \mathrm{P}<0.05$ vs. treatment with Ang II.

practice $(17,18)$. However, extensive research has focused on their potential effects on cardiovascular diseases. Due to their inhibitory effects on the mevalonate pathway and particularly FPPS, they now act as a tool used to investigate the role of FPPS in various diseases $(19,20)$. FPPS has been implicated in the development of various cardiovascular diseases, including myocardial and vascular remodeling and endothelial dysfunction in SHRs (12-16). The inhibition of FPPS by alendronate or its knockdown by RNA interference has been shown to prevent cardiac hypertrophy and fibrosis both in vivo and in vitro (12-14). Moreover, the cardiac-specific overexpression of FPPS has been shown to induce cardiac hypertrophy and heart failure in mice (15). In addition, in a recent study of ours, we demonstrated that the inhibition of FPPS prevented NE-induced fibrotic responses, including cell proliferation, the hydroxyproline content and CTGF protein expression in SHR-VSMCs (16), indicating that FPPS may function as a potent regulator of vascular remodeling.

The exaggerated VSMC activities and excessive ECM protein accumulation induced by Ang II are thought to be key to the devolepment of vascular remodeling (3-9). A number of pharmacological agents, such as anti-thrombotics, antiplatelet agents, angiotensin-converting enzyme inhibitors, as well as mechanical and cellular approaches have been used in an attempt to attenuate this pathophysiological process $(21,22)$. A growing numbers of studies in the literature have demonstrated that bisphosphonates may play an important role in inhibiting the development of atherosclerosis and neointimal hyperplasia 

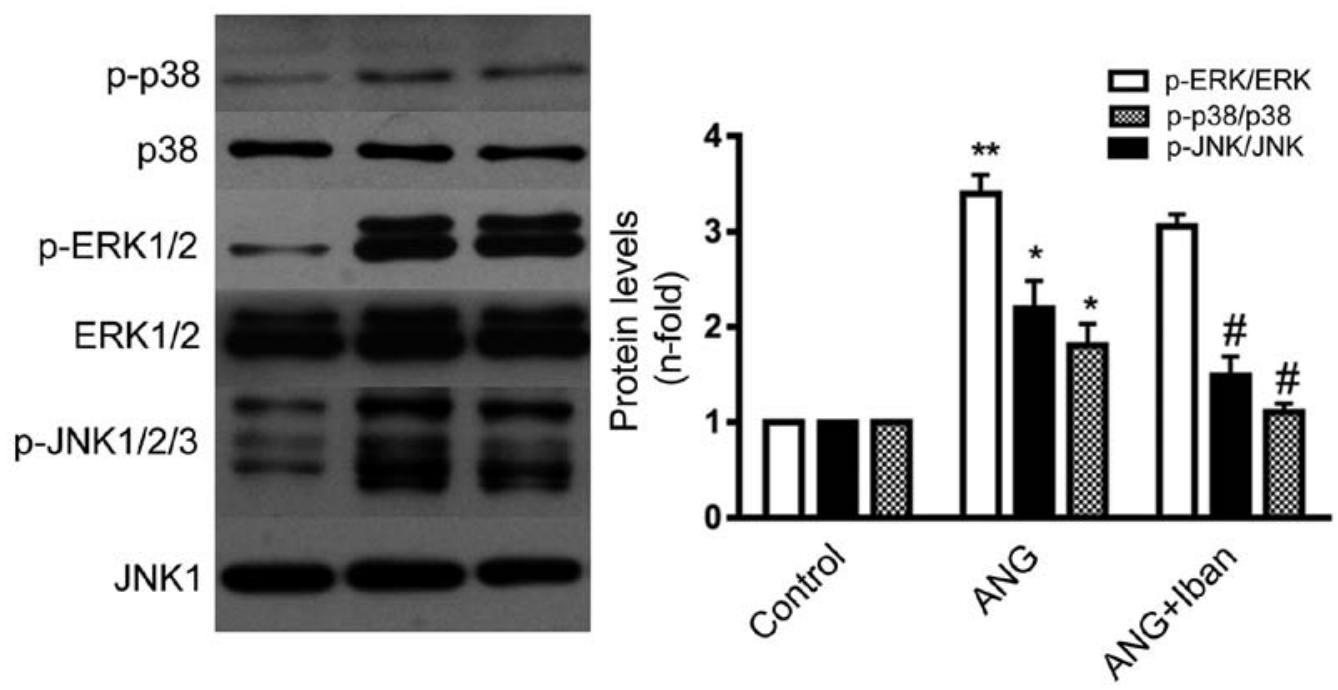

Figure 6. Ibandronate sodium (Iban) diminishes mitogen-activated protein kinase (MAPK) activation in angiotensin II (Ang II)-stimulated vascular smooth muscle cells (VSMCs). Cells were pre-treated for $24 \mathrm{~h}$ with Iban $(10 \mu \mathrm{M})$, and then stimulated with $0.1 \mu \mathrm{M}$ Ang II for 15 min. Left panel shows a representative western blot of phosphorylated p38 (p-p38), phosphorylated extracellular signal-regulated kinase (p-ERK1/2) and phosphorylated c-Jun N-terminal kinase (JNK) (p-JNK1/2/3) and p38, ERK and JNK (used as controls). Right panel shows data, presented as the means \pm SEM of 3 experiments. The ratio of p-ERK1/2or p-p38 or p-JNK1/2/3 is set to ' 1 ' for control group. ${ }^{*} \mathrm{P}<0.05$ and ${ }^{* *} \mathrm{P}<0.01$ vs. control; ${ }^{*} \mathrm{P}<0.05$ vs. treatment with Ang II.

in animal models. These effects are thought to be mediated by the transient systemic inactivation of monocytes and macrophages $(23,24)$. However, in a balloon-injured rat carotid artery model, both the systemic and local delivery of zoledronate was shown to prevent intimal hyperplasia (25). Additionly, in a rabbit carotid anastomosis model, zoledronic acid (ZA), a third generation of N-BPs, was demonstrated to inhibit neointimal hyperplasia and decrease VSMC intensity, suggesting that it may have a direct inhibitory effect on VSMCs (26). Indeed, etidronate, another bisphosphonate, has been reported to exert an inhibitory effect on the growth of VSMCs from SHRs (27). Similarly, in cultured rat VSMCs, ZA has also been reported to inhibit the proliferation, adhesion and migration of VSMCs without the induction of necrosis or apoptosis (28). Recently, ZA was reported to inhibit the growth of stimulated human arotic smooth muscle cells, as well as their proliferation adhesion and migration, but had no effect on quiescent cells. The addition of GGOH significantly reversed the ZA-mediated alteration in cellular viability and RAP1A/B prenylation, suggesting that ZA modulates the mevalonate pathway and inhibits the prenylation of GTPase binding proteins (29). Taken together, the above data point out that BPs or N-BPs may play an important role in vascular remodeling by modulating cell signaling, including the prenylation of small signaling proteins (e.g., Ras, Rac, Rab and Rho) through the mevalonate pathway. However, the underlying mechanisms through which BPs or N-BPs inhibit VSMC activity and prevent intimal hyperplasia in animal models induced by FPPS were not elucidated in the above-mentioned studies.

In our previous study, we reported that Iban inhibited NE-induced fibrotic responses in SHR-VSMCs through the modulation of the Ras kinase and p38 pathways (16). In the present study, we further demonstrated that Iban also inhibits fibrotic responses in VSMCs induced by Ang II. As shown in Fig. 1 and in our previous study (16), Iban alone did not influence cell proliferation and CTGF expression in quiescent
VSMCs, but markedly reduced the Ang II-induced increase in cell proliferation and CTGF expression (Figs. 2 and 3). Besides, Ang II upregulated FPPS in the cultured VSMCs. To determine whether the anti-fibrotic effects of Iban depend on the modulation of FPPS, analogues or antagonists of the mevalonate pathway were used in this study. The results revealed that addition of $\mathrm{GGOH}$, but not that of $\mathrm{FOH}$, partly reversed the anti-fibrotic effects of Iban (Fig. 3). In addition, GGTI-286, a specific inhibitor of GGTase I, but not FTI-276 (a selective inhibitor of farnesyltransferase), mimicked the anti-fibrotic effects of Iban (Fig. 3). Since RhoA is a geranylgeranylated protein (30), our results suggest that RhoA signaling is involved in this process.

In a previous study, it was clearly demonstrated that pretreatment with RhoA and ROCK inhibitors diminished the Ang II-induced overexpression of CTGF, indicating that Ang II increases the production of CTGF in VSMCs through the RhoA/ROCK pathway (7). In the present study, the inhibition of FPPS with Iban diminished the Ang II-induced RhoA activation in cultured VSMCs, suggesting that FPPS may be involved in the modulation of Ang II-induced CTGF production through the RhoA/ROCK pathway. This is consistent with the results of other studies on the effects of in vivo and in vitro alendronate treatment with hypertrophic response induced by Ang II in cultured neonatal ventricular myocytes and animal models $(12,13)$. However, the levels of isoprenoid intermediates, such as FOH or GGOH were not measured after the suppression of FPPS. The knockdown of FPPS by RNA interference in VSMCs is another critical issue to be confirmed in view of the role of FPPS in Ang II-mediated vascular remodeling. Besides, the in vivo effect of the vascular-specific interference of FPPS in vascular remodeling needs to be validated in animal models. These limitations require further investigation.

It is well known that MAPKs are a widely distributed group of enzymes. The consisting of 3 isoforms (ERK, p38 and JNK) and play an important role in Ang II-mediated vascular 
remodeling $(7,8,31)$. To determine whether Iban modulates the Ang II-induced overexpression of CTGF by altering the activity of the MAPK pathway, VSMCs were pre-treated with the specific inhibitor of the MAPK pathway prior to stimulation with Ang II. As shown in Fig. 5, both the JNK and p38 MAPK inhibitor diminished the Ang II-induced production of CTGF. This is consistent with the findings of a pregvious study on the effects of HMG-CoA reductase inhibitors (statins) on the modulation of Ang II-mediated vascular responses (7). Furthermore, as previously described (7) and shown in Fig. 6 in the present study, treatment with Ang II triggered the phosphorylation of all 3 MAPKs. However, only the phosphorylation of JNK and p38 was diminished by treatment with Iban. These data indicate that JNK and p38 MAPK may be involved in the Iban-mediated inhibition of CTGF overexpression induced by Ang II in VSMCs.

In conclusion, in this study, we provide evidence that FPPS expression is elevated in Ang II-stimulated VSMCs. The ihibibition of FPPS by Iban attenuates the Ang II-induced increase in cell proliferation and CTGF expression in VSMCs, and the underlying mechanisms, at least in part, involve the modulation of RhoA activity, and the p38 and JNK pathways.

\section{Acknowledgements}

This study was supported by the grants from the Natural Science Foundation of Zhejiang province (Project for Young Scientists, no. LQ13H020004).

\section{References}

1. Rizzoni D, Muiesan ML, Porteri E, De Ciuceis C, Boari GE, Salvetti M, Paini A and Rosei EA: Vascular remodeling, macroand microvessels: Therapeutic implications. Blood Press 18: 242-246, 2009

2. Inoue $\mathrm{T}$ and Node $\mathrm{K}$ : Molecular basis of restenosis and novel issues of drug-eluting stents. Circ J 73: 615-621, 2009.

3. Touyz RM and Schiffrin EL: Signal transduction mechanisms mediating the physiological and pathophysiological actions of angiotensin II in vascular smooth muscle cells. Pharmacol Rev 52: 639-672, 2000.

4. Fan WH, Pech M and Karnovsky MJ: Connective tissue growth factor (CTGF) stimulates vascular smooth muscle cell growth and migration in vitro. Eur J Cell Biol 79: 915-923, 2000.

5. Oemar BS, Werner A, Garnier JM, Do DD, Godoy N, Nauck M, März W, Rupp J, Pech M and Lüscher TF: Human connective tissue growth factor is expressed in advanced atherosclerotic lesions. Circulation 95: 831-839, 1997.

6. Rupérez M, Lorenzo O, Blanco-Colio LM, Esteban V, Egido J and Ruiz-Ortega M: Connective tissue growth factor is a mediator of angiotensin II-induced fibrosis. Circulation 108 : 1499-1505, 2003.

7. Rupérez M, Rodrigues-Díez R, Blanco-Colio LM, Sánchez-López E, Rodríguez-Vita J, Esteban V, Carvajal G, Plaza JJ, Egido J and Ruiz-Ortega M: HMG-CoA reductase inhibitors decrease angiotensin II-induced vascular fibrosis: Role of RhoA/ROCK and MAPK pathways. Hypertension 50: 377-383, 2007.

8. Ohtsu H, Mifune M, Frank GD, Saito S, Inagami T, Kim-Mitsuyama S, Takuwa Y, Sasaki T, Rothstein JD, Suzuki H, et al: Signal-crosstalk between Rho/ROCK and c-Jun NH2-terminal kinase mediates migration of vascular smooth muscle cells stimulated by angiotensin II. Arterioscler Thromb Vasc Biol 25: 1831-1836, 2005.

9. Kobayashi N, Nakano S, Mita S, Kobayashi T, Honda T, Tsubokou Y and Matsuoka H: Involvement of Rho-kinase pathway for angiotensin II-induced plasminogen activator inhibitor-1 gene expression and cardiovascular remodeling in hypertensive rats. J Pharmacol Exp Ther 301: 459-466, 2002.
10. Roskoski R Jr: Protein prenylation: A pivotal posttranslational process. Biochem Biophys Res Commun 303: 1-7, 2003.

11. Li L, Hu SJ, Dong HT, Kang L, Chen NY and Fang YQ: Alterations in gene expression of series key enzymes in mevalonic acid pathway detected by RNA array in spontaneously hypertensive rats. Chin J Pathophysiol 24: 54-59, 2008.

12. Ye Y, Hu SJ and Li L: Inhibition of farnesylpyrophosphate synthase prevents angiotensin II-induced hypertrophic responses in rat neonatal cardiomyocytes: Involvement of the RhoA/Rho kinase pathway. FEBS Lett 583: 2997-3003, 2009.

13. Ye Y, Mou Y, Bai B, Li L, Chen GP and Hu SJ: Knockdown of farnesylpyrophosphate synthase prevents angiotensin II-mediated cardiac hypertrophy. Int J Biochem Cell Biol 42: 2056-2064, 2010.

14. Li L, Chen GP, Yang Y, Ye Y, Yao L and Hu SJ: Chronic inhibition of farnesyl pyrophosphate synthase attenuates cardiac hypertrophy and fibrosis in spontaneously hypertensive rats. Biochem Pharmacol 79: 399-406, 2010.

15. Yang J, Mou Y, Wu T, Ye Y, Jiang JC, Zhao CZ, Zhu HH, Du CQ, Zhou $\mathrm{L}$ and $\mathrm{Hu}$ SJ: Cardiac-specific overexpression of farnesyl pyrophosphate synthase induces cardiac hypertrophy and dysfunction in mice. Cardiovasc Res 97: 490-499, 2013.

16. Du CQ, Yang L, Yang J, Han J, Hu XS, Wu T and Hu SJ: Inhibition of farnesyl pyrophosphate synthase prevents norepinephrineinduced fibrotic responses in vascular smooth muscle cells from spontaneously hypertensive rats. Hypertens Res 37: 26-34, 2014.

17. Owens JM, Fuller K and Chambers TJ: Osteoclast activation: Potent inhibition by the bisphosphonate alendronate through a nonresorptive mechanism. J Cell Physiol 172: 79-86, 1997.

18. Papapoulos SE: Ibandronate: A potent new bisphosphonate in the management of postmenopausal osteoporosis. Int J Clin Pract 57: 417-422, 2003.

19. van Beek E, Pieterman E, Cohen L, Löwik C and Papapoulos S: Farnesyl pyrophosphate synthase is the molecular target of nitrogen-containing bisphosphonates. Biochem Biophys Res Commun 264: 108-111, 1999.

20. Kavanagh KL, Guo K, Dunford JE, Wu X, Knapp S, Ebetino FH, Rogers MJ, Russell RG and Oppermann U: The molecular mechanism of nitrogen-containing bisphosphonates as antiosteoporosis drugs. Proc Natl Acad Sci USA 103: 7829-7834, 2006.

21. Lefkovits $\mathbf{J}$ and Topol EJ: Pharmacological approaches for the prevention of restenosis after percutaneous coronary intervention. Prog Cardiovasc Dis 40: 141-158, 1997.

22. Kiernan TJ, Yan BP, Cruz-Gonzalez I, Cubeddu RJ, Caldera A, Kiernan GD and Gupta V: Pharmacological and cellular therapies to prevent restenosis after percutaneous transluminal angioplasty and stenting. Cardiovasc Hematol Agents Med Chem 6: 116-124, 2008.

23. Ylitalo R, Oksala O, Ylä-Herttuala S and Ylitalo P: Effects of clodronate (dichloromethylene bisphosphonate) on the development of experimental atherosclerosis in rabbits. J Lab Clin Med 123: 769-776, 1994.

24. Danenberg HD, Golomb G, Groothuis A, Gao J, Epstein H, Swaminathan RV, Seifert P and Edelman ER: Liposomal alendronate inhibits systemic innate immunity and reduces in-stent neointimal hyperplasia in rabbits. Circulation 108: 2798-2804, 2003.

25. Wu L, Zhu L, Shi WH, Yu B and Cai D: Zoledronate inhibits intimal hyperplasia in balloon-injured rat carotid artery. Eur J Vasc Endovasc Surg 41: 288-293, 2011.

26. Güzeloğlu M, Gül M, Reel B, Yürekli I, Aykut K and Hazan E: The effects of zoledronic acid on neointimal hyperplasia: A rabbit carotid anastomosis model. Anadolu Kardiyol Derg 11: 93-100, 2011.

27. Su JZ, Fukuda N, Kishioka H, Hu WY and Kanmatsuse K: Etidronate influences growth and phenotype of rat vascular smooth muscle cells. Pharmacol Res 46: 7-13, 2002.

28. Wu L, Zhu L, Shi WH, Zhang J, Ma D and Yu B: Zoledronate inhibits the proliferation, adhesion and migration of vascular smooth muscle cells. Eur J Pharmacol 602: 124-131, 2009.

29. Albadawi H, Haurani MJ, Oklu R, Trubiano JP, Laub PJ, Yoo HJ and Watkins MT: Differential effect of zoledronic acid on human vascular smooth muscle cells. J Surg Res 182: 339-346, 2013.

30. Van Aelst L and D'Souza-Schorey C: Rho GTPases and signaling networks. Genes Dev 11: 2295-2322, 1997.

31. Miyata Y and Nishida E: Distantly related cousins of MAP kinase: Biochemical properties and possible physiological functions. Biochem Biophys Res Commun 266: 291-295, 1999. 